\title{
COMPORTAMIENTO VISCOELÁSTICO DE PULPA DE MEMBRILLO EN FUNCIÓN DE LA CONCENTRACIÓN DE SÓLIDOS SOLUBLES ${ }^{1}$
}

\author{
Afonso Mota RAMOS ${ }^{2 *}$, Albert IBARZ ${ }^{3}$
}

\begin{abstract}
RESUMEN
El conocimiento de las propiedades viscoelásticas de los alimentos es muy útil en el diseño y predicción de la estabilidad de muestras almacenadas. El trabajo tuvo como objetivos caracterizar las propiedades viscoelásticas de las muestras de pulpa de membrillo a diferentes concentraciones de sólidos solubles. El estudio reológico del pulpa de membrillo fue realizado en el rango de concentraciones de 12,3 a $28^{\circ} \mathrm{Brix}$ a la temperatura de $20^{\circ} \mathrm{C}$. Las medidas viscoelásticas fueron realizadas con un reômetro Haake Rheostress RS100 y fue seleccionado el barrido de frecuencia entre 0,1 a $100 \mathrm{~Hz}$. Los resultados demostraron que para la muestra de $28^{\circ} \mathrm{Brix}$ la pulpa se comportó como un semi-sólido en todo el rango de frecuencia estudiada. Para la concentración de $24^{\circ} \mathrm{Brix}$, a bajas frecuencias, la pulpa se comportó como un semi-líquido ( $G$ ” > G') mientras que en frecuencias más altas se comportó como semi-sólido (G’> G"). Para la pulpa de 16 e $20^{\circ}$ Brix hubo el predominio del carácter viscoso sobre el carácter elástico. Para la pulpa de $12,3^{\circ}$ Brix no fue posible determinar los valores G' e G”. Se observó todavía que la viscosidad compleja del pulpa de membrillo aumenta con la concentración para todo el rango de frecuencias estudiadas.

Palabras clave: pulpa de membrillo, viscoelasticidad, viscosidad compleja.
\end{abstract}

\section{RESUMO}

COMPORTAMENTO VISCOELÁSTICO DE POLPA DE MARMELO EM FUNÇÃO DA CONCENTRAÇÃO DE SÓLIDOS SOLÚVEIS. O conhecimento das propriedades viscoelásticas dos alimentos é muito útil no desenho e na predição da estabilidade de amostras armazenadas. O trabalho teve como objetivo caracterizar as propriedades viscoelásticas de amostras de polpa de marmelo a diferentes concentrações de sólidos solúveis. O estudo reológico da polpa de marmelo foi realizado na faixa de concentração de 12,3 a $28^{\circ}$ Brix à temperatura de $20^{\circ} \mathrm{C}$. As medidas viscoelásticas foram realizadas com um reômetro Haake Rheostress RS100 e foi selecionado um varrido de freqüência (frequency sweep) entre 0,1 e $100 \mathrm{~Hz}$. Os resultados demonstraram que para a amostra de $28^{\circ} \mathrm{Brix}$ a polpa se comportou como um semi-sólido ( $\mathrm{G}^{\prime}>\mathrm{G}^{\prime}$ ) em toda faixa de freqüência estudada. Para a concentração de $24^{\circ} \mathrm{Brix}$, a baixas freqüências, a polpa se comportou com um semi-líquido ( $G^{\prime \prime}>G^{\prime}$ ), enquanto que em freqüências mais altas se comportou como semi-sólido (G'> G”). Para as concentrações de 16 e $20^{\circ}$ Brix houve predomínio do caráter viscoso sobre o caráter elástico. Para a polpa de $12,3^{\circ}$ Brix não foi possível determinar os valores G' e G”. A viscosidade complexa da polpa de marmelo aumentou com a concentração de sólidos solúveis para toda a faixa de freqüência estudada.

Palavras-chave: polpa de marmelo, viscoelasticidade, viscosidade complexa.

\section{1 - INTRODUCCIÓN}

Muchos alimentos incluyen en su composición una matriz sólida más o menos elástica y una fase líquida que aporta un elemento viscoso a la estructura total. Estos alimentos presentan un comportamiento reológico que combina características propias de los sólidos elásticos y de los líquidos, y se denomina viscoelástico [5]. El conocimiento de las propiedades viscoelásticas es muy útil en el diseño y predicción de la estabilidad de muestras almacenadas $[11,15]$.

Para un sistema disperso, como es el caso de derivados líquidos de frutas, que fluye con característica no newtoniana el cambio estructural es inmediato y no detectable.

En general los alimentos viscoelásticos no exhiben un comportamiento lineal y para el estudio de la viscoelasticidad deben definirse las condiciones experimentales para que

\footnotetext{
$\overline{{ }^{1} \text { Recebido para publicação em 9/6/2005. Aceito para publicação em }}$ 23/1/2006 (001549)

${ }^{2}$ Departamento de Tecnologia de Alimentos. Universidade Federal de Viçosa -36.570-000 - Viçosa (MG).E-mail:amramos@ufv.br

${ }^{3}$ Departamento de Tecnología de Aliments. Universitat de Lleida - UdL -Lleida -Espanha.E-mail: aibarz@tecal.udl.es
}

se establezcan las relaciones entre las variables esfuerzo, deformación y tiempo, de manera que se mantenga la linealidad de respuesta viscoelástica.

A través del crecimiento del esfuerzo se puede caracterizar la viscoelasticidad de los alimentos mediante el estudio de la variación del esfuerzo cortante con el tiempo a una velocidad de deformación fija hasta alcanzar un equilibrio. Esta técnica es semejante a la caracterización tixotrópica y el análisis comparativo de los reogramas obtenidos permite una caracterización práctica de los ensayos.

También se puede caracterizar mediante la relajación del esfuerzo, donde se aplica en el alimento una deformación en cizalla simple y se observa la variación del esfuerzo con el tiempo de cizalla.

Otra forma de caracterizar la viscoelasticidad de los fluidos alimenticios es mediante los ensayos reológicos dinámicos donde se aplica de forma oscilatoria una pequeña deformación o velocidad de deformación sobre un fluido y la amplitud de la respuesta del esfuerzo cortante y del ángulo de fase entre el esfuerzo cortante y la deformación se mide. Este ensayo corresponderá al estado de viscoelasticidad lineal si el esfuerzo es linealmente proporcional a la deformación aplicada y si la respuesta del esfuerzo es 
en forma de una onda sinusoidal [14]. En el caso de un sólido elástico esta onda está en fase con la velocidad de deformación aplicada. Para un líquido viscoso ideal existe un desfase de $90^{\circ}$ entre ellos. En fluidos viscoelásticos el ángulo de desfase está comprendido entre 0 y $90^{\circ}$.

Este tipo de ensayo permite determinar la proporción entre el componente elástico y viscoso de un material y cuantificar en qué medida se comporta como sólido o como líquido. De hecho se necesita conocer el valor de una serie de funciones viscoelásticas.

Se definen dos propiedades reológicas, el módulo de almacenamiento, $\mathrm{G}^{\prime}$, que representa la componente elástica del producto, y el módulo de pérdida, $\mathrm{G}^{\prime \prime}$, que representa su carácter viscoso y pueden expresarse de la siguiente manera:

$$
\begin{aligned}
& G^{\prime}=G^{*} \cos \delta=\left(\frac{\tau_{0}}{\gamma_{0}}\right) \cos \delta \\
& G^{\prime \prime}=G^{*} \operatorname{sen} \delta=\left(\frac{\tau_{o}}{\gamma_{0}}\right) \operatorname{sen} \delta
\end{aligned}
$$

en la que $\tau_{\mathrm{o}} \mathrm{y} \gamma_{\mathrm{o}}$ son, respectivamente, las amplitudes de las ondas del esfuerzo y de la deformación y $\delta$ es el ángulo de desfasaje. Si el fluido es puramente elástico, $\delta=0^{\circ}$ y $\mathrm{G}^{*}=\mathrm{G}^{\prime}$, $\mathrm{G}^{\prime \prime}=0$. En un fluido viscoso ideal $\delta=90^{\circ}$ y $\mathrm{G}^{\prime \prime}=\mathrm{G}^{*}, \mathrm{G}^{\prime}=0$.

Otra importante función viscoelástica es el módulo de viscosidad compleja, $\eta^{*}$, que se define como [2]:

$$
\left|\eta^{*}\right|=\frac{G^{*}}{\Omega}=\left|\left(\eta^{\prime}\right)^{2}+\left(\eta^{\prime \prime}\right)^{2}\right|^{1 / 2}
$$

donde la parte real, la viscosidad dinámica $\eta^{\prime}$ representa la componente viscosa en fase entre el esfuerzo y la velocidad de deformación, y la parte imaginaria $\eta^{\prime \prime}$ es la componente elástica o desfasada. Los parámetros $\mathrm{G}^{\prime \prime}$ y $\eta^{\prime}$ pueden considerarse como una contribución viscosa asociada con una disipación de energía hecha sobre el fluido, mientras $\mathrm{G}^{\prime} \mathrm{y}$ $\eta^{\prime \prime}$ una contribución asociada con la habilidad del fluido de almacenar energía [3].

Estas funciones viscoelásticas han sido usadas para caracterizar algunos tipos de alimentos tales como, dispersiones de pectinas [2], proteinas de cereales [11], mantequilla [19], gel de surimi [16] y gel de proteína de pescado [12].

Este trabajo tuvo como objetivos caracterizar las propiedades viscoelásticas de la pulpa de membrillo a diferentes concentraciones en sólidos solubles.

\section{2 - MATERIAL Y MÉTODOS}

La muestra de la pulpa de membrillo, con concentración de sólidos solubles de $12,3^{\circ} \mathrm{Brix}$, fue suministrada por una industria transformadora de frutas ubicadas en la Provincia de Lleida (España). Esta pulpa fue producida en esta industria a través de tecnología de procesamiento aséptico. Las muestras fueron suministradas en bolsas asépticas de $3 \mathrm{~kg}$.

\section{1 - Caracterización físico-química de las muestras}

La muestra de pulpa de membrillo fue caracterizada por las determinaciones físico-químicas que se indican en los próximos subapartados. Todas estas determinaciones se han realizado con cinco repeticiones.

Previamente, las muestras fueron preparadas de acuerdo con las especificaciones de cada método.

\subsection{1 - Concentraciones en sólidos solubles}

El contenido en sólidos solubles se determinó según el método oficial de [1] en un refratómetro Atago RX-1000 a $20^{\circ} \mathrm{C}$, expresándose el resultado en ${ }^{\circ}$ Brix.

\subsection{2 - Acidez total titulável}

La acidez total se determinó utilizando el método oficial de análisis de zumos y otros derivados [17]. Los resultados se expresan en gramos de ácido cítrico/100 mL de muestra.

\subsection{3 - pH}

Se determinó según el método oficial de [1], en un pHmetro digital Crisson Micro-pH 2000.

\subsection{4 - Azúcares}

La determinación de los azúcares se realizó mediante cromatografia líquida de alta resolución (HPLC). El cromatógrafo utilizado está formado por los siguientes componentes: un desgasificador en línea Knauer Desgasser, un controlador de gradiente Waters ${ }^{\mathrm{TM}} 600$, un detector de absorbancia Water 486 Detector Absorbance, un detector de índice de refracción Waters 410 Differencial Refractometer y un integrador que recoge las señales del detector Waters 748 Data Module. Se utilizó la precolumna PLHi-Plex Pb Guard de 50 mm x 7,7 mm y una columna PL Hi-Plex Pb de $300 \mathrm{~mm} \mathrm{x} \mathrm{7,7} \mathrm{mm.}$ Ambas formadas a base de resina monodispersa para análisis de carbohidratos (relleno monodisperso de estireno sulfonado y copolímero divinilbenzeno de tamaño de partícula $8 \mu \mathrm{m}$. Los azúcares que fueron determinados son sacarosa, glucosa y fructosa.

Las condiciones de trabajo fueron

Fase móvil: agua bidestilada

Flujo: $1 \mathrm{~mL} / \mathrm{min}$

Temperatura de la coluna: $85^{\circ} \mathrm{C}$

Temperatura de detección en el refractómetro: $31^{\circ} \mathrm{C}$

Presión de trabajo: 500 psi 
La metodología seguida fue la del patrón externo. Se construyó una curva patrón para cada uno de los tres azúcares, inyectando distintas diluciones, de concentración conocida, del azúcar correspondiente.

Este método se utilizó como alternativo del método oficial, ya que se consideró que presenta excelentes resultados y que además es un método simple y rápido [6].

\subsection{5 - Fibra}

El contenido en fibras se determinó por un método enzimático-gravimétrico [1], basado en la acción secuencial de las enzimas $\alpha$-amilasa, proteasa y amiloglucosidasa, en condiciones de $\mathrm{pH}$ y temperatura específicas para cada uno de ellos. Luego las muestras son centrifugadas. El residuo se lava con agua destilada, por dos veces, agitando y centrifugando cada vez. Los sobrenadantes se deben guardar y reservar. Después se lava, se agita y se centrifuga el residuo, primero con etanol 96\% y después con acetona, desechando el sobrenadante. Se secan los tubos con el residuo en estufa a $105^{\circ} \mathrm{C}$ durante una noche y se pesa (FFI).

$\mathrm{Al}$ volumen de sobrenadante guardado se añaden cuatro veces su volumen de etanol $96 \%$ a $60^{\circ} \mathrm{C}$ y se deja en reposo durante $1 \mathrm{~h}$ a temperatura ambiente. Se filtra a vacío esa muestra en una placa porosa de vidrio previamente tarada y que contiene $250 \mathrm{mg}$ de celite y se desecha el filtrado. Se lava el residuo con etanol $96 \%$, etanol $80 \%$ y acetona, y se desecha el filtrado. Se secan las placas en estufa a $105^{\circ} \mathrm{C}$ durante una noche y se pesa (FFS).

Para la obtención del blanco se realiza todo el procedimiento anterior sin añadir la muestra. Se hacen los análisis de proteína por el método Kjeldahl y contenido en cenizas de los residuos FFI y FFS. También se determina la humedad de la muestra a $105^{\circ} \mathrm{C}$ durante $24 \mathrm{~h}$. Los resultados se expresan en porcentaje en peso sobre la muestra seca.

\subsection{6 - Pulpa en suspensión}

Se determinó centrifugando $50 \mathrm{~g}$ de muestra durante 15 min a 15.000 rpm (29.000 G), expresándose los resultados en porcentaje en peso de la pulpa separada respecto a la muestra inicial [4].

\subsection{7 - Pectinas}

La determinación de las pectinas totales se realizó de acuerdo con el método oficial recomendado por la Internacional Federation of Fruit Juice Producers [10]. Se procesa la extracción de las sustancias pécticas de las muestras primero con etanol $96 \%$ y en caliente, y después del residuo resultante con etanol 63\% y en caliente. Se efectúa un lavado del precitado con $\mathrm{NaOH}$ y se filtra. El filtrado resultante constituye la fracción de pectinas totales. Este filtrado se trata con carbazol y ácido sulfúrico para dar la reacción colorimétrica correspondiente. Se cuantifica la fracción de las pectinas totales con un espectrofotómetro Philips PU 8700 mediante la absorbancia de la muestra a 525 nm. Se construye una curva de calibrado utilizando ácido galacturónico como patrón. Los resultados se expresan en mg de ácido galacturónico por kg de muestra.

\subsection{8 - Actividad del agua}

La actividad de agua se determinó utilizando el medidor de actividad de agua Novasina Termoconstanter RTD-200 TH-2 a temperatura de $25^{\circ} \mathrm{C}$.

\section{2 - Caracterización viscoelástica}

La caracterización viscoelástica fue realizada en las muestras de pulpa industrial de membrillo en concentraciones de 12,$3 ; 16 ; 20 ; 24$ y $28^{\circ} \mathrm{Brix}$.

Para la obtención de las diferentes concentraciones en sólidos solubles de la pulpa de membrillo se ha dispuesto de un equipo de evaporación a vacío Rotavapor Resona Technics Labo Rota a una temperatura de trabajo de $50^{\circ} \mathrm{C}$. Las muestras obtenidas de la concentración a vacío han sufrido un congelamiento rápido en un congelador de placas con el objetivo de evitar la formación de grandes cristales de hielo y danificaciones en el tejido celular del vegetal. Luego las muestras han sido almacenadas en congelador a una temperatura de $-18^{\circ} \mathrm{C}$.

Las medidas viscoelásticas han sido realizadas con un reómetro Haake Rheostress RS100 [7]. El sistema sensor seleccionado fue el cono-placa $\mathrm{C} 35 / 4^{\circ}$. En este estudio, previo a la medición, todas las muestras se quedaron en reposo durante 15 min para permitir la relajación de las mismas.

Para cada muestra y concentración se realizaron ensayos previos para elegir el valor de la amplitud (amplitud sweep) para el que las variaciones de las funciones viscoelásticas dinámicas en función de la frecuencia se encuentren en rango lineal. Este valor de amplitud del esfuerzo cortante obtenido fue de $350 \mathrm{~Pa}$. Así se ha seleccionado el barrido de frecuencia (frequency sweep) entre 0,1 a $100 \mathrm{~Hz}$ para la ejecución de los trabajos experimentales.

La temperatura de las muestras fue mantenida a través de un sistema de atemperación, el cual consiste en un circuito de agua que permite mantener la temperatura a $20 \pm 0,1^{\circ} \mathrm{C}$. La entrada de la deformación y la salida de los datos de esfuerzo cortante fue automáticamente controlado por el programa REOSTRESS RS Versión 2.08 P instalado en un ordenador PC conectado con el reómetro Haake. Las medidas fueron realizadas en tres repeticiones.

\section{3 - RESULTADOS}

\section{1 - Caracterización físico-química}

Los resultados de la caracterización físico-química de la pulpa de membrillo están recogidos en la Tabla 1.

\section{2 - Caracterización viscoelástica}

Los resultados de los ensayos reológicos experimen- 
TABLA 1 - Valores de las características físico-químicas determinados en la muestra de la pulpa de membrillo

\begin{tabular}{lc}
\hline Variable & Pulpa de membrillo \\
\hline Sólidos solubles $\left({ }^{\circ}\right.$ Brix $)$ & 12,3 \\
Actividad del agua & 0,983 \\
$\mathrm{pH}$ & 3,59 \\
Acidez & 0,40 \\
(g de ác. cítrico/100 mL) & 53,9 \\
Contenido en pulpa $(\%)$ & 220,70 \\
Pectinas & 4,52 \\
(mg de ac. galacturónico/kg) & 24,2 \\
Fibra $(\%$ en peso) & 61,44 \\
Glucosa $(\mathrm{g} / \mathrm{L})$ & 19,45 \\
Fructosa $(\mathrm{g} / \mathrm{L})$ & \\
Sacarosa(g/L)
\end{tabular}

tales oscilatorios se expresan en términos del módulo de almacenamiento (G') y del módulo de pérdida (G”). Si G'>G', el material alimenticio exhibe un comportamiento de un semi-sólido, esto es, tendrá más característica elástica que viscosa; y, por tanto, si G">G', el material se comporta como un semi-líquido y la energía usada en la deformación del material está asociada con la disipación viscosa.

Los valores obtenidos de la caracterización, son semejantes a los encontrados en trabajos de [8, 9, 13,18].

La Figura 1 presenta los valores del módulo de almacenamiento y del módulo de pérdida a la temperatura de

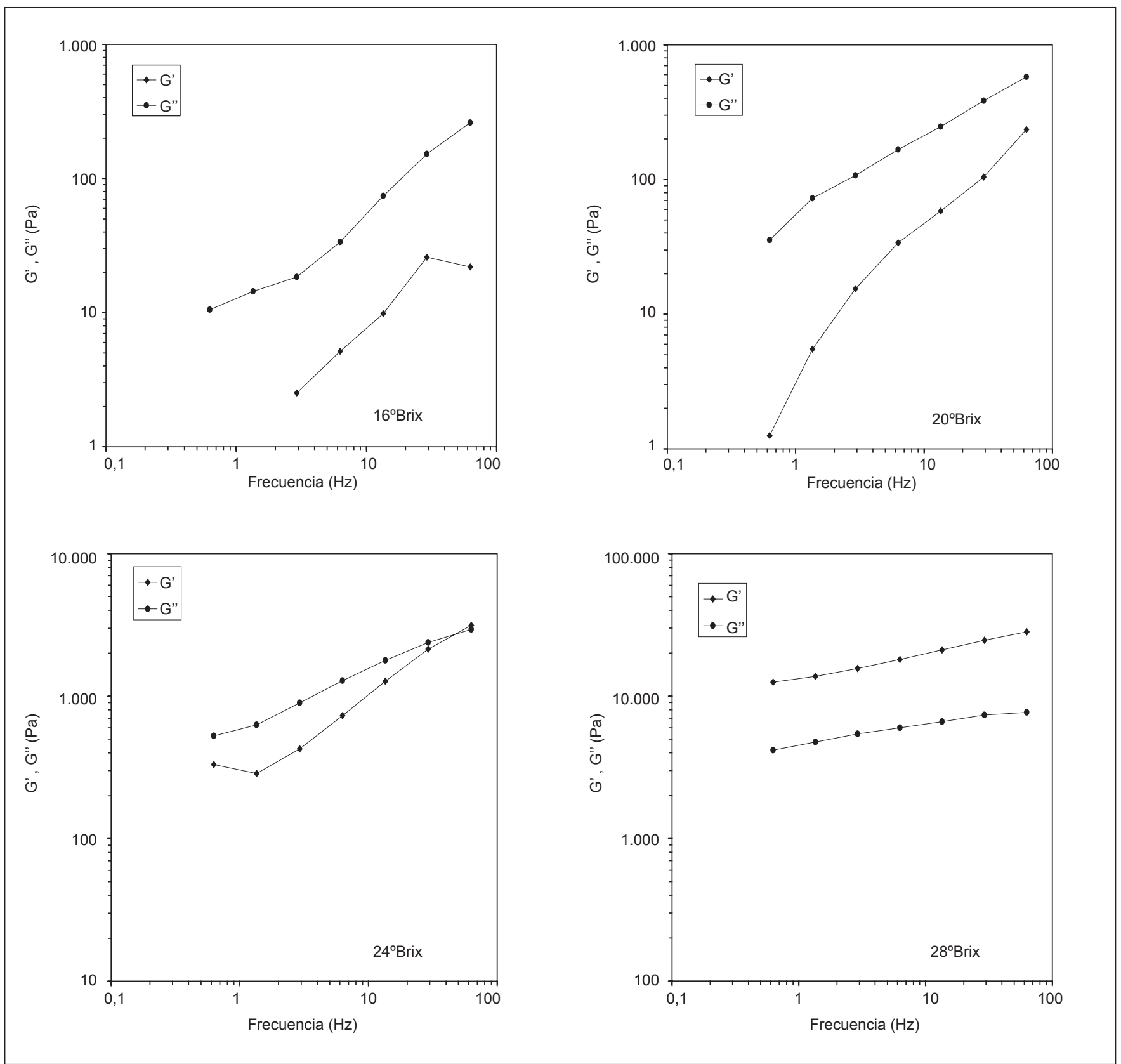

FIGURA 1 - Valores del módulo de almacenamiento (G') y del módulo de pérdida (G”) en función de la frecuencia de la pulpa de membrillo a la temperatura de $20^{\circ} \mathrm{C}$ en concentraciones de $16,20,24$ y $28^{\circ} \mathrm{Brix}$ 
$20^{\circ} \mathrm{C}$ en función de la frecuencia para las muestras de pulpa de membrillo a las distintas concentraciones en sólidos solubles ensayadas. Se puede observar que existen diferencias cuantitativas en el espectro en las magnitudes de los parámetros viscoelásticos de la pulpa de membrillo. Así, para la muestra más concentrada a $28^{\circ}$ Brix, apenas hubo alteraciones de los valores G' y G” con la frecuencia, y el pulpa se comporta como un semi-sólido en todo el rango de frecuencia estudiada, donde predomina el carácter elástico sobre el viscoso (G'>G"). Para la muestra de concentración de $24^{\circ}$ Brix, a bajas frecuencias el pulpa se comporta como un semi-líquido (G”>G') y predomina el carácter viscoso sobre el elástico, mientras que se observan menores diferencias entre G' y G" a medida que aumenta la frecuencia hasta alcanzar un predominio del carácter elástico sobre el carácter viscoso (G'>G”). Esta tendencia ha sido observada para las muestras de concentraciones de 16 y $20^{\circ} \mathrm{Brix}$, a pesar de que se comportan como semi-líquido para todo el rango de frecuencia estudiada, donde predomina el carácter viscoso sobre el carácter elástico. Este tipo de comportamiento ha sido observado por otros investigadores en trabajos con dispersiones de pectinas [3] y con mayonesa [15].

Las magnitudes del módulo de almacenamiento aumentan con el incremento de la concentración en sólidos solubles de la pulpa, lo que indica una tendencia a un comportamiento más predominante de un alimento semi-sólido (Figura 1). Este mayor valor para el módulo de almacenamiento a concentraciones más elevadas puede ser atribuido a su mayor contenido en pectina que implica la formación de un gel más fuerte, así como también la formación de una estructura más compleja de moléculas de cadena larga y de partículas fuertemente solvatadas formando una red más compacta.

La Figura 2 muestra las magnitudes absolutas de las viscosidades complejas, $\left|\eta^{*}\right|$ para las distintas concentraciones en sólidos solubles de la pulpa de membrillo a la temperatura de $20^{\circ} \mathrm{C}$ en función de la frecuencia. Se puede observar que la viscosidad compleja aumenta con la concentración en sólidos solubles para todo el rango de frecuencias estudiadas $(0,63$ a $62,8 \mathrm{~Hz}$ ) por el hecho de que una concentración más alta implica una formación de una estructura más compacta.

Los valores de la tangente del ángulo de fase, ángulo $\delta$, denominado como la función de pérdidas $(\tan \delta)=\mathrm{G}^{\prime} / \mathrm{G}^{\prime}$, para el pulpa de membrillo a las distintas concentraciones estudiadas están representados en la Figura 3. La tan $\delta$ es una medida adimensional que compara la cantidad de energía perdida durante un ensayo oscilatorio con la cantidad de energía almacenada durante este periodo, e indica si predomina la propiedad elástica o viscosa. Se puede apreciar que la pendiente para la concentración de $20^{\circ} \mathrm{Brix}$ es mayor que para $24^{\circ} \mathrm{Brix}$, mientras que la pendiente para la concentración de $28^{\circ} \mathrm{Brix}$ es cercana a cero, lo que confirma para esta última concentración la mayor predominancia de propiedades elásticas. Para la muestra a concentración de $16^{\circ}$ Brix, debido a su carácter predominantemente viscoso (Figura 1) y que presenta bajos valores de viscosidades complejas (Figura 2), presenta una pendiente distinta de las demás muestras de membrillo (Figura 3).

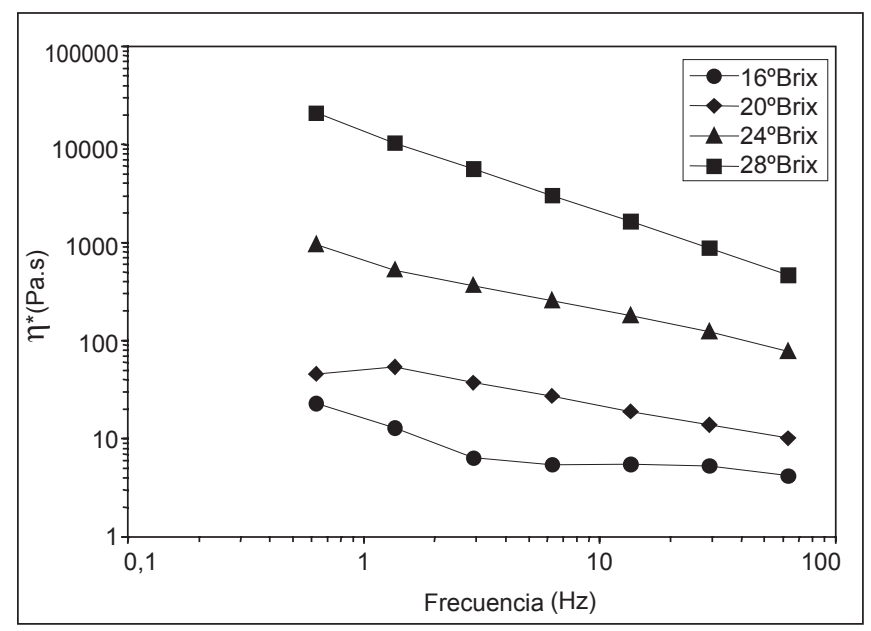

FIGURA 2 - Efecto de la concentración de la pulpa de membrillo a la temperatura de $20^{\circ} \mathrm{C}$ sobre la viscosidad compleja ( $\left.\eta^{*}\right)$

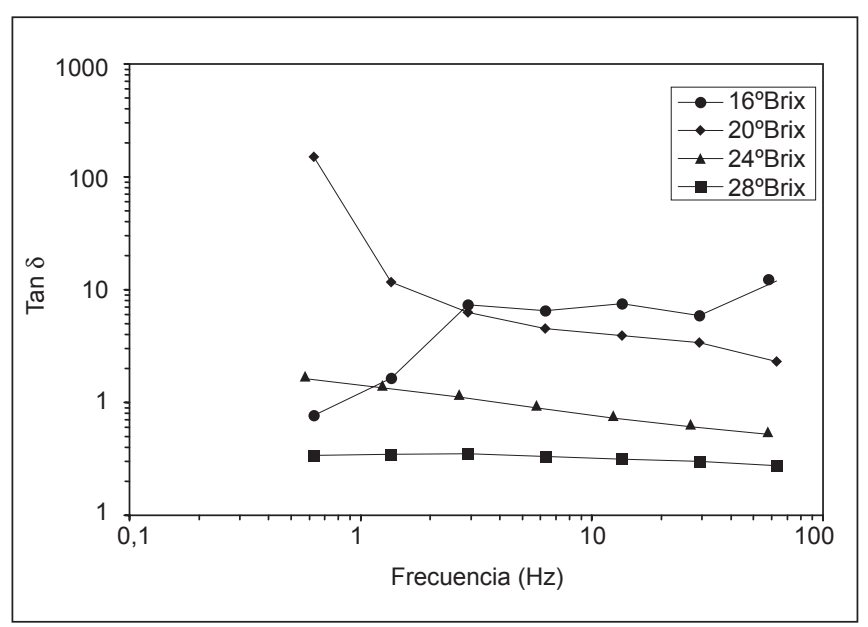

FIGURA 3 - Efecto de la concentración de la pulpa de membrillo a la temperatura de $20^{\circ} \mathrm{C}$ sobre la función de pérdidas $(\tan \delta)$

\section{4 - CONCLUSÕES}

La pulpa de membrillo a la concentración en sólidos solubles de $28^{\circ} \mathrm{Brix}$ tiene un comportamiento de un material viscoelastico e de este modo se comporta como un alimento semi-sólido en el rango de frecuencia estudiada, donde predomina su carácter elástico. Esto puede ser atribuido a su mayor contenido en pectina que implica la formación de un gel más fuerte. La pulpa de concentración de $24^{\circ} \mathrm{Brix}$ a bajas frecuencias se comporta como un alimento semi-líquido, mientras que en frecuencias más elevadas se comporta como semi-sólido. Las concentraciones de 12,3 y $16^{\circ}$ Brix predomina en el pulpa el carácter viscoso, así que se comporta como un alimento semi-líquido.

\section{5 - REFERÊNCIAS BIBLIOGRÁFICAS}

[1] A.O.A.C. Official Methods of analysis, Association of Official Analytical Chemists. Washington, D.C., 1990.

[2] DA SILVA, J.A.L; RAO, M.A. Rheology of structure 
development in high-methoxyl pectin/sugar systems, Food Technol., p. 70-73, October, 1995.

[3] DA SILVA, J.A.L.; GONÇALVES, M.P; RAO, M.A. Viscoelastic behaviour of mixtures of locust bean gum and pectin dispersions, J. Food Eng., v. 18, p. 211-228, 1993.

[4] DURÁN, L.; JIMÉNEZ, G. Evolución de las propiedades reológicas del pulpa de albaricoque durante la operación de concentración, Rev. Agroquím. Tecnol. Aliment, v. 20(2), p. 209-219, 1980.

[5] FIZMAN, S.M.; COSTELL, E.; DURÁN, L. Medida del comportamiento reológico de alimentos sólidos, II. Métodos fundamentales, Rev. Agroquím. Tecnol. Aliment., v. 23(3), p. 303-309, 1983.

[6] GARZA, S.G.; GINER, J.; MARTÍN, O.; COSTA, E.; IBARZ, A. Colour, sugars and HMF evolution during thermal treatment of apple juice, Food Sci. and Technol. Int, v. 2 (2), p. 101-110, 1996.

[7] HAAKE, RheoStress-RS100. HAAKE Mess-Technic GmbH u. Co. Karluhe, Germany, 1995.

[8] HERNÁNDEZ, E.; CHEN, C.S.; JOHNSON, J.; CARTER, R.D. Viscosity changes in orange juice after ultrafiltration and evaporation, J. Food Eng, v. 25(3), p. 387-396, 1995.

[9] IBARZ, A.; ORTIZ, M.J. Reología de zumos de melocotón. Influencia del contenido en pulpa, Alimentación, Equipos y Tecnología, p. 81-86, octubre, 1993.

[10] IFFP. International Federation of Fruit Juice Producers Methods, Analysen-analyces, Zug, Switzerland, 1984.
[11] KOKINI, J.L.; PLUTCHOK, G.J. Viscoelastic properties of semisolid foods and their biopolymeric components, Food Technol., p. 89-95, March, 1987.

[12] LEE, H.G.; LANIER, T.C.; HAMANN, D.D. Chemically induced covalent crosslinks affect thermo-rheological profiles of protein gels, J. Food Sc., v. 62 (1), p. 29-32, 1997.

[13] LOZANO, J.E.; IBARZ, A. Thixotropic behaviour of concentrated fruit pulps, Lebensm-wiss u. - Technol, v. 27(1), p.16-18, 1994.

[14] MA, L.; BARBOSA-CÁNOVAS, G.V. Rheological properties of food gums and food gum mixtures, Rev. Esp. Cienc. Tecnol. Aliment., v. 33(2), p. 133-163, 1993.

[15] MA, L.; BARBOSA-CÁNOVAS, G.V. Rheological characterization of mayonnaise. Part II: Flow and viscoelastic properties at different oil and xanthan gum concentrations, J. Food Eng., v. 25, p. 409-425, 1995.

[16] MA, L.; GROVE, A.; BARBOSA-CÁNOVAS, G.V. Viscoelastic characterization of surimi gel: Effect of setting and starch, J. Food Sci., v. 61(5), p. 881-883, 1996.

[17] MAPA. Métodos oficiales de análisis. Tomo I. Secretaria General Técnica, Ministerio de Agricultura, Pesca, y Alimentación, Madrid, 1993.

[18] RAMOS, A. M.; IBARZ, A. Tixotropy of orange concentrate and quince puree, Journal of Texture studies, v. 29, p. 313-324, 1998.

[19] SHUKLA, A.; RIZVI, S.S.H. Viscoelastic properties of butter, J. Food Sci., v. 60(5), p. 902-905, 1995. 